\title{
Expression and co-expression analyses of TMPRSS2, a key element in COVID-19
}

\author{
Francesco Piva ${ }^{1} \cdot$ Berina Sabanovic $^{1} \cdot$ Monia Cecati $^{1} \cdot$ Matteo Giulietti ${ }^{1}$ \\ Received: 29 June 2020 / Accepted: 28 October 2020 / Published online: 27 November 2020 \\ (C) Springer-Verlag GmbH Germany, part of Springer Nature 2020
}

\begin{abstract}
The ACE2 receptor is, so far, the best-known host factor for SARS-CoV-2 entry, but another essential element, the TMPRSS2 protease, has recently been identified. Here, we have analysed TMPRSS2 expression data in the lung correlating them with age, sex, diabetes, smoking habits, exposure to pollutant and other stimuli, in order to highlight which factors might alter TMPRSS2 expression, and thus impact the susceptibility to infection and COVID-19 prognosis. Moreover, we reported TMPRSS2 polymorphisms affecting its expression and suggested the ethnic groups more prone to COVID-19. Finally, we also highlighted a gender-specific co-expression between TMPRSS2 and other genes related to SARS-CoV-2 entry, maybe explaining the higher observed susceptibility of infection in men. Our results could be useful in designing potential prevention and treatment strategies regarding the COVID-19.
\end{abstract}

Keywords COVID-19 $\cdot$ TMPRSS2 $\cdot$ Age $\cdot$ Co-expression $\cdot$ Gender

\section{Introduction}

The outbreak of coronavirus disease 2019 (COVID-19) in Wuhan, China, is rapidly spreading worldwide. In fact, as of October 2020, over 1 million deaths and 37 million positive cases have been reported globally [1]. Spike protein 3D structures of severe acute respiratory syndrome-coronavirus (SARS-CoV), responsible for the 2003 outbreak, and of the current SARS-CoV-2, resulted to be sufficiently similar to interact with the same host cell targets [2]. Indeed, recent studies reported the involvement of angiotensin-converting enzyme 2 (ACE2) and the transmembrane serine protease 2 (TMPRSS2) also in SARS-CoV-2 infection [3]. Specifically, virus entry through the cell surface receptor ACE2 requires proteolytic cleavage and activation of the spike proteins by TMPRSS2 protease. Accordingly, the TMPRSS2 inhibitor

Supplementary Information The online version contains supplementary material available at https://doi.org/10.1007/s10096-02004089-y.

Francesco Piva

f.piva@univpm.it

1 Department of Specialistic Clinical and Odontostomatological Sciences, Polytechnic University of Marche, Ancona, Italy camostat mesylate blocked SARS-CoV-2 entry and might represent a treatment option [3].

\section{Analysis of TMPRSS2 expression data}

Recent epidemiological data indicate that COVID-19 showed higher rates of critical illness and deaths in men, in smokers, in diabetics and in older people [4-7]. TMPRSS2 is mainly expressed in the lung, salivary gland, thyroid, gastrointestinal tract, pancreas, kidney and liver, according to RNA and protein expression data available at Human Protein Atlas (HPA) database (Fig. 1). Notably, it is also expressed in many male tissues, such as ductus deferens, epididymis, seminal vesicle and prostate, but it has very low expression, or it is absent in the testis and in all female tissues, including the breast, ovary, fallopian tube, endometrium and placenta. This data may suggest a role of TMPRSS2 tissue distribution in the higher SARS-CoV-2 infection rate and severity in men. However, additional studies are necessary to validate many other possible reasons for the observed gender difference, including risk factors and protection of female hormones. In addition, TMPRSS2 gene is upregulated by androgenic hormones also in the lungs [8]. Very recently, Asselta et al. analysed publicly available gene expression datasets, reporting that TMPRSS2 expression is higher in bronchial epithelial cells of males than females, whereas the 

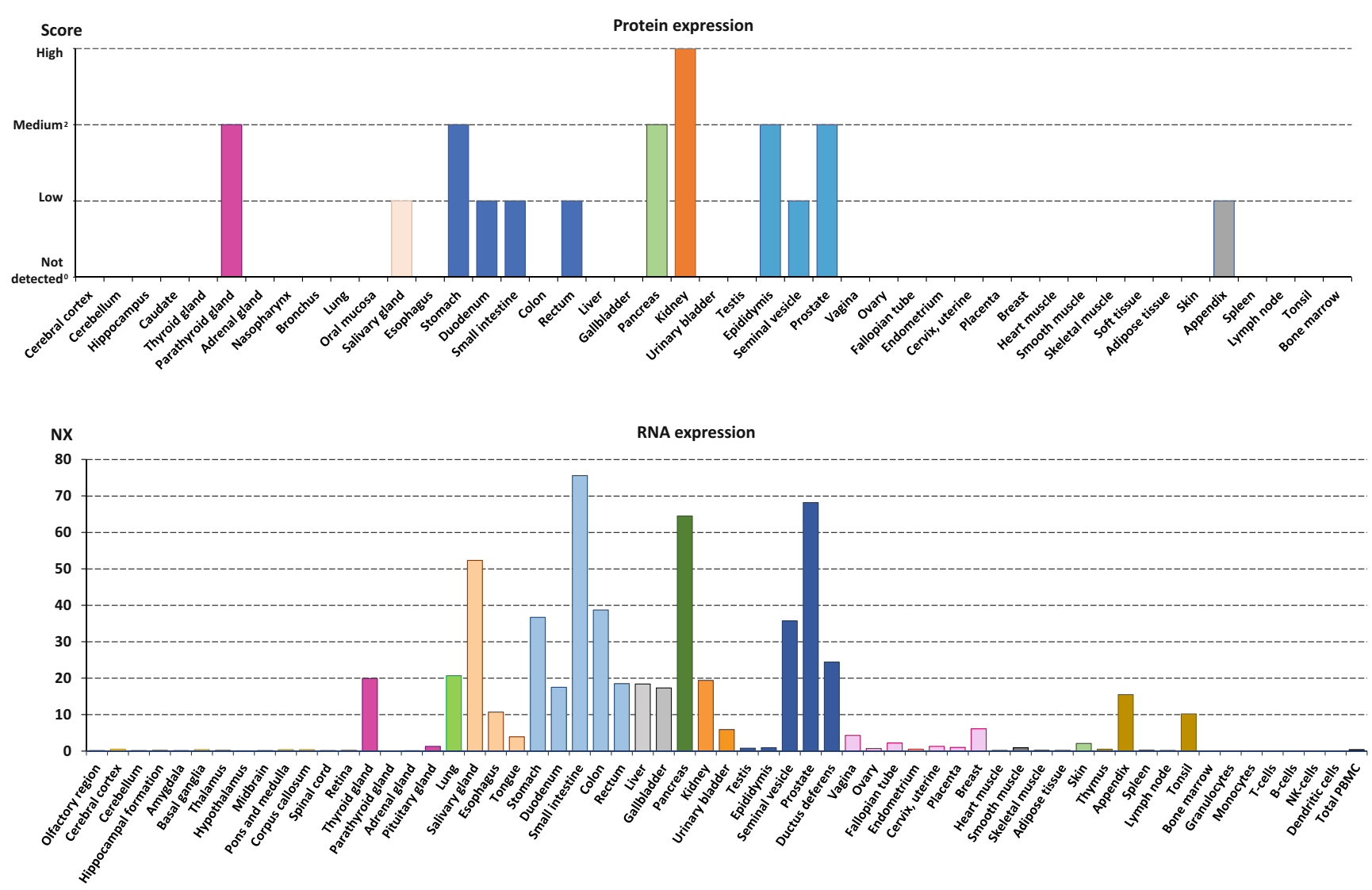

Fig. 1 Protein (top) and RNA (bottom) expression of TMPRSS2 in human tissues, according to Human Protein Atlas (HPA) database (https://www. proteinatlas.org/ENSG00000184012-TMPRSS2/tissue)

expression in lung was similar [9]. Here, we further investigate the effect of gender on TMPRSS2 expression and extend these analyses also to the effect of age, smoking behaviour, infections and pollutants in the lung, nasal and bronchial tissues and cell lines (Supplementary Table S1).

In three microarray datasets (GSE40419, GSE19804 and GSE10072) from NCBI GEO, no statistically significant differences have been identified in the TMPRSS2 expression in lung tissues by stratifying for gender or smoke habit status, but, unexpectedly, there is a weak decreasing age-related trend (Supplementary Figs. S1, S2, and S3). In particular, significant differences between under/over 65-year-old people ( $p=$ $0.044)$ and between under/over 75-year-old people $(p=0.012)$ have been observed. However, there are no sex- or agespecific differences in the TMPRSS2 expression in lung tissues, according to GSE117261 and RNA-seq datasets from GTEx (https://www.gtexportal.org/) data. In nasal epithelium samples (GSE8987), cigarette smoking significantly increases TMPRSS2 expression $(p=0.014)$. Recently, this expression increase in smoker subjects as compared to non-smokers has also been observed by western blots analysis in the lung [10] and by microarray analysis in small airway epithelium [11]. Since TMPRSS2 expression seems unexpectedly to decrease with increasing age, other factors, including diabetes, lower levels of androgens, pollutants or a genetic predisposition, could be relevant to explain the observed epidemiological trends in this outbreak (Supplementary Table S1). Regarding these factors, we found that TMPRSS2 is two-fold higher expressed $(p=0.00046)$ in pancreatic beta-cells of type 2 diabetes (T2D) subjects than controls (GSE20966). Although not in the lungs, this result suggests a possible explanation of why diabetes mellitus is an important risk factor for hospitalization and death in COVID-19 patients. Moreover, the TMPRSS2 expression is significantly increased in normal bronchial epithelial cells infected by 2009 pandemic H1N1 influenza virus (GSE48466) and, likewise, in lung fibroblasts after exposure to vanadium pentoxide (GSE5339). In addition, TMPRSS2 gene expression slightly increased following exposition to benzo[a]pyrene diol epoxide (BPDE) in normal lung fibroblasts (GSE19510). On the other hand, TMPRSS2 levels decrease following inhaled corticosteroids therapy in bronchial biopsies from allergic asthma subjects (GSE15823) or after exposure to 9-nm silica nanoparticles in lung epithelial cells (GSE53700) (Supplementary Table S1). This evidence suggests that concomitance with other infections, and some workplace pollutants could make lung cells more susceptible to the SARS-CoVs. Moreover, our analysis shows that TMPRSS2 expression is not altered by interferon 
Fig. 2 Violin plots of TMPRSS rs2070788 (left) and rs383510 (right) eQTLs in the lung, as reported in GTEx portal. All genotypes (along with the number of tested subjects), the resulting median TMPRSS expression levels in the lung and the $p$ values for the association between the gene expression and the genotypes are shown. In particular, GTEx generated $p$ values for each variantgene pair by testing the alternative hypothesis that the slope of a linear regression model between genotype and expression deviates from 0. Data source: GTEx Analysis Release V8 rs2070788

chr21_41470061_G_A_b38
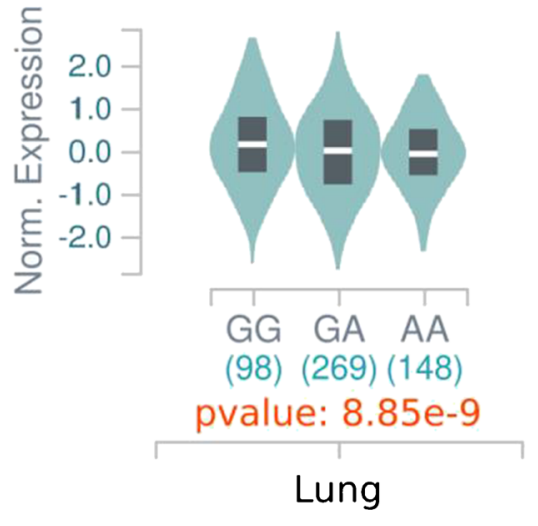

rs383510

chr21_41486440_T_C_b38
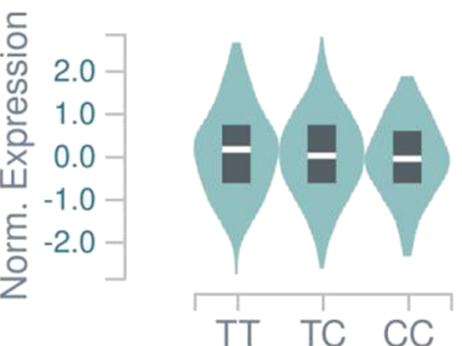

(113) (251) (151)

pvalue: $1.2 \mathrm{e}-8$

Lung $\begin{array}{lllllll}0.163 & -0.015 & -0.043 \quad \text { Median values } 0.127 & -0.005 & -0.053\end{array}$
(IFN) treatment in lung epithelial cells (GSE5542). Accordingly, it has recently been reported that interferon inflammation does not modulate TMPRSS2 expression but upregulates ACE2 expression [12]. Instead, TMPRSS2 expression in airway epithelia is highly upregulated by IL-13 $[12,14]$, a highly expressed cytokine in plasma of COVID19 patients that require ventilation [13]. Furthermore, IL-13 is also involved in allergic airway type 2 inflammation, asthma and chronic obstructive pulmonary disease (COPD) [12, 14], thus suggesting a further mechanism for COVID-19 susceptibility and severity.

\section{Polymorphisms}

Expression quantitative trait loci (eQTLs) are sequence variations, mainly single-nucleotide polymorphisms (SNPs), affecting the expression levels of genes [15]. Here, we investigated GTEx and QTLbase databases (http://mulinlab.org/ qtlbase/index.html) about TMPRSS2 gene expression, and we reported 19 increasing and 24 decreasing eQTLs (Supplementary Table S2). Overall, they are experimentally confirmed to affect TMPRSS2 expression with a NES score (normalized effect size, see Supplementary Table S2 for details) up to $+/-0.12$, a level of alteration often sufficient to induce phenotypic changes. Indeed, among variations reported in Table S2, rs2070788 GG or the rs383510 TT genotypes (both with +0.11 NES, $p=8.85 \mathrm{e}-09$ and $p=1.20 \mathrm{e}-08$, respectively, Fig. 2) are shown to increase TMPRSS2 expression in lung tissue and the susceptibility to severe A(H1N1) and $\mathrm{A}(\mathrm{H} 7 \mathrm{~N} 9)$ influenza [16]. Notably, QTLbase reports that, in the lung, rs2070788 GG increases expression also of MX1, a gene involved in the antiviral response, which expression level is higher in COVID-19 patients and reduced with age [17]. Our analyses indicates that also TMPRSS2 expression decreases with age, and interestingly, these genes are positively co-expressed in males and negatively in females ( $r=-0.288, p=0.0036$ in females; $r=0.402, p=1.046 \mathrm{e}-8$ in males).

Moreover, according to the NCBI dbSNP database (www.ncbi. nlm.nih.gov/snp/), Americans, Mexicans, Europeans and South Asians have a higher rs2070788 G allele frequency than Africans, Native Hawaiians, African American descents and East Asians. Regarding rs383510, Europeans, Estonians and South Asians have a higher $\mathrm{T}$ allele frequency than East Asians, Koreans and Africans, so the former could be more prone to infection. However, since the observed expression variations are enough to increase influenza susceptibility, it is possible that many other eQTLs in TMPRSS2 gene could alter the susceptibility to SARS-CoV-2 infection. For example, some new eQTLs [12] and missense SNPs altering TMPRSS2 expression and function have been recently suggested to influence SARS-CoV-2 entry and

Table 1 ACE2 co-expression levels with other host factors in the lung

\begin{tabular}{ll}
\hline Gene pairs & Pearson's co-expression \\
\hline ACE2 TMPRSS2 & $r=0.4485(p=1.187 \mathrm{e}-15)$ \\
ACE2 CTSB & $r=0.3985(p=2.126 \mathrm{e}-12)$ \\
ACE2 FURIN & $r=0.3142(p=5.142 \mathrm{e}-8)$ \\
ACE2 CTSL & $r=0.2599(p=0.000007893)$ \\
ACE2 BSG (CD147) & $r=0.2494(p=0.00001858)$ \\
ACE2 CD209 & $r=0.04397(\mathrm{NS})$ \\
ACE2 TMPRSS11D & $r=0.03035(\mathrm{NS})$ \\
ACE2 TMPRSS11A & $r=-0.1256(p=0.03308)$ \\
ACE2 CLEC4M & $r=-0.1481(p=0.01185)$ \\
\hline
\end{tabular}

We used UCSC Xena Functional Genomics Explorer (https:// xenabrowser.net/) to analyse correlation of GTEx data in lung tissues using Pearson's correlation test. Pearson's $r$ values range between -1 and 1 . Positive values mean positive correlation between the expression levels of the gene pair; on the contrary, negative values show an inverse correlation; $r$ values close to 0 mean no correlation. NS not significant 
Table 2 Gender-specific TMPRSS2 co-expression levels with other host factors in the lung

\begin{tabular}{lll}
\hline TMPRSS2 VS & Females & Males \\
\hline ACE2 & $r=0.4403(p=4.61 \mathrm{e}-6)$ & $r=0.4551(p=5.42 \mathrm{e}-11)$ \\
BSG (CD147) & $r=0.1506(p=0.1349)$ & $r=0.6561(p=1.730 \mathrm{e}-24)$ \\
FURIN & $r=0.3618(p=2.18 \mathrm{e}-4)$ & $r=0.7446(p=2.01 \mathrm{e}-34)$ \\
CTSB & $r=0.1714(\mathrm{NS})$ & $r=0.6195(p=2.74 \mathrm{e}-21)$ \\
CTSL & $r=-0.00164(\mathrm{NS})$ & $r=0.5658(p=2.75 \mathrm{e}-17)$ \\
CD209 & $r=-0.2632(p=0.0082)$ & $r=0.2559(p=0.0004)$ \\
TMPRSS11A & $r=-0.1363(\mathrm{NS})$ & $r=-0.1638(p=0.0247)$ \\
TMPRSS11D & $r=-0.1200(\mathrm{NS})$ & $r=-0.04262(\mathrm{NS})$ \\
CLEC4M & $r=-0.2493(p=0.0124)$ & $r=-0.03108(\mathrm{NS})$ \\
\hline
\end{tabular}

See Table 1 for details

COVID-19 severity $[9,18,19]$. However, in order to identify causal host genetic risk factors for COVID-19, large-scale genome-wide association studies (GWAS) and functional assays are needed. Moreover, it should be taken into account that, in addition to the possible genetic predisposition, infection rates and treatment outcomes could be influenced by different socioeconomic status and other ethnic disparities [20].

\section{Sex-specific TMPRSS2 co-expression with other host factors for viral entry}

We performed correlation analyses in GTEx RNA-seq data in lung tissues in order to identify the co-expression levels among TMPRSS2, ACE2 and other coronavirus host factors. Our analyses indicated that the highest degree of coexpression in normal lung tissues is between ACE2 and TMPRSS2 $(r=0.4485, p=1.187 \mathrm{e}-15$, Table 1$)$. This strong association may explain the predominant role of TMPRSS2 in priming ACE2 compared to other proteases in SARS-CoVs. Moreover, since men are more prone to being affected by COVID-19, we also investigated the sex-specific expression correlation of TMPRSS2 with the other host factors involved in SARS-CoV entry. Our analysis shows that some gene coexpressions are maintained in both males and females, but others are present only in men (Table 2). Notably, TMPRSS2 is highly and positively co-expressed with CD147 receptor and FURIN, CD209, cathepsin L (CTSL) and $\mathrm{B}$ (CTSB) proteases in men, but not in women, while the co-expression with ACE2 is similarly high in both males and females. These results indicate that, in males, the co-expression of these proteases and CD147, a newly discovered SARS-CoV-2 receptor [11], may enhance the host priming activity on SARS-CoV spike proteins. In turn, it may result in a higher viral entry, which explains the higher infection risk and illness severity in men than women. Interestingly, since co- expressed genes share a similar regulatory network involving common transcription factors and microRNAs, further studies should be carried out in order to clarify the gender-specific molecular regulation of these host factors for viral entry.

\section{Conclusions}

In conclusion, our analyses of the TMPRSS2 expression data could suggest a link between the risk of SARS-CoV-2 infection and the COVID-19 severity with genetic predispositions, age, gender, diabetes, air pollutants and smoking. Our preliminary results could lead to further researches for validations in wider cohorts and for experimental assessments to characterize the underlying molecular mechanisms. Also, they could be useful for the identification of new druggable targets, besides IL-13 and androgens, responsible of induction of TMPRSS2 expression. In this regard, treatment strategies to block SARSCoV-2 entry involving TMPRSS2 inhibition have been proposed, including camostat, nafamostat, bromhexine [21] and androgen deprivation therapies [22]. Since we have found that some factors could increase TMPRSS2 expression, and therefore to affect COVID-19 susceptibility and severity, these should be taken into account during the definition of a personalized therapy. For example, the presence of specific SNPs or lifestyle habits could help to indirectly estimate the TMPRSS2 expression levels in the lungs for patient stratification. In fact, subjects with high TMPRSS2 levels would benefit more from TMPRSS2 inhibitors. In addition, it was recently hypothesized that extracellular vesicles can enhance SARS-CoV-2 spreading to uninfected cells by transferring ACE2 [23]. Since also TMPRSS2 protein is present in salivary vesicles [24] and it could act similarly, the utility of these molecules as severity biomarkers should be investigated. Finally, since in males we have found strong co-expression levels of TMPRSS2 with CD147, FURIN, CTSB and CTSL, these could be targeted along with TMPRSS2 in order to enhance the treatments for COVID-19 male patients.

Availability of data and material Not applicable

\section{Compliance with ethical standards}

Conflict of interest The authors declare that they have no conflict of interest.

Ethics approval Not applicable

Consent to participate Not applicable

Consent for publication Not applicable

Code availability Not applicable 


\section{References}

1. WHO. Coronavirus disease (COVID-19) situation report. Weekly Epidemiological Update (12 October 2020). https://www.who.int/ docs/default-source/coronaviruse/situation-reports/20201012weekly-epi-update-9.pdf

2. Vankadari N, Wilce JA (2020) Emerging WuHan (COVID-19) coronavirus: glycan shield and structure prediction of spike glycoprotein and its interaction with human CD26. Emerg Microbes Infect 9:601-604. https://doi.org/10.1080/22221751.2020. 1739565

3. Hoffmann M, Kleine-Weber H, Schroeder S, Kruger N, Herrler T, Erichsen S, Schiergens TS, Herrler G, Wu NH, Nitsche A, Muller MA, Drosten C, Pohlmann S (2020) SARS-CoV-2 cell entry depends on ACE2 and TMPRSS2 and is blocked by a clinically proven protease inhibitor. Cell 181:271-280.e8. https://doi.org/10.1016/ j.cell.2020.02.052

4. Cai H (2020) Sex difference and smoking predisposition in patients with COVID-19. Lancet Respir Med 8:e20. https://doi.org/10. 1016/S2213-2600(20)30117-X

5. Fadini GP, Morieri ML, Longato E, Avogaro A (2020) Prevalence and impact of diabetes among people infected with SARS-CoV-2. J Endocrinol Invest 43:867-869. https://doi.org/10.1007/s40618020-01236-2

6. Ge H, Wang X, Yuan X, Xiao G, Wang C, Deng T, Yuan Q, Xiao $X$ (2020) The epidemiology and clinical information about COVID-19. Eur J Clin Microbiol Infect Dis 39:1011-1019. https://doi.org/10.1007/s10096-020-03874-z

7. Guan WJ, Ni ZY, Hu Y, Liang WH, Ou CQ, He JX, Liu L, Shan H, Lei CL, Hui DSC, Du B, Li LJ, Zeng G, Yuen KY, Chen RC, Tang CL, Wang T, Chen PY, Xiang J, Li SY, Wang JL, Liang ZJ, Peng YX, Wei L, Liu Y, Hu YH, Peng P, Wang JM, Liu JY, Chen Z, Li G, Zheng ZJ, Qiu SQ, Luo J, Ye CJ, Zhu SY, Zhong NS, China Medical Treatment Expert Group for Covid-19 (2020) Clinical characteristics of coronavirus disease 2019 in China. N Engl J Med 382:1708-1720. https://doi.org/10.1056/NEJMoa2002032

8. Mikkonen L, Pihlajamaa P, Sahu B, Zhang FP, Janne OA (2010) Androgen receptor and androgen-dependent gene expression in lung. Mol Cell Endocrinol 317:14-24. https://doi.org/10.1016/j. mce.2009.12.022

9. Asselta R, Paraboschi EM, Mantovani A, Duga S (2020) ACE2 and TMPRSS2 variants and expression as candidates to sex and country differences in COVID-19 severity in Italy. Aging (Albany NY). 12: 10087-10098. 10.18632/aging.103415

10. Maremanda KP, Sundar IK, Li D, Rahman I (2020) Age-dependent assessment of genes involved in cellular senescence, telomere, and mitochondrial pathways in human lung tissue of smokers, COPD, and IPF: Associations With SARS-CoV-2 COVID-19 ACE2TMPRSS2-Furin-DPP4 Axis. Front Pharmacol. 11:584637. https://doi.org/10.3389/fphar.2020.584637

11. Matusiak M, Schürch CM (2020) Expression of SARS-CoV-2 entry receptors in the respiratory tract of healthy individuals, smokers and asthmatics. Respir Res. 21:252. https://doi.org/10.1186/ s12931-020-01521-X

12. Sajuthi SP, DeFord P, Li Y, Jackson ND, Montgomery MT, Everman JL, Rios CL, Pruesse E, Nolin JD, Plender EG, Wechsler ME, Mak ACY, Eng C, Salazar S, Medina V, Wohlford EM, Huntsman S, Nickerson DA, Germer S, Zody MC, Abecasis G, Kang HM, Rice KM, Kumar R, Oh S, Rodriguez-Santana J, Burchard EG, Seibold MA (2020) Type 2 and interferon inflammation regulate SARS-CoV-2 entry factor expression in the airway epithelium. Nat Commun. 11:5139. https://doi.org/10.1038/s41467-020-18781-2

13. Donlan AN, Young M, Petri WA, Abhyankar M (2020) IL-13 predicts the need for mechanical ventilation in COVID-19 Patients. medRxiv 2020.06.18.20134353. https://doi.org/10.1101/ 2020.06.18.20134353

14. Kimura H, Francisco D, Conway M, Martinez FD, Vercelli D, Polverino F, Billheimer D, Kraft M (2020) Type 2 inflammation modulates ACE2 and TMPRSS2 in airway epithelial cells. J Allergy Clin Immunol. 146:80-88.e8. https://doi.org/10.1016/j. jaci.2020.05.004

15. Cookson W, Liang L, Abecasis G, Moffatt M, Lathrop M (2009) Mapping complex disease traits with global gene expression. Nat Rev Genet. 10:184-194. https://doi.org/10.1038/nrg2537

16. Cheng Z, Zhou J, To KK, Chu H, Li C, Wang D, Yang D, Zheng S, Hao K, Bossé Y, Obeidat M, Brandsma CA, Song YQ, Chen Y, Zheng BJ, Li L, Yuen KY (2015) Identification of TMPRSS2 as a susceptibility gene for severe 2009 pandemic $\mathrm{A}(\mathrm{H} 1 \mathrm{~N} 1)$ influenza and A(H7N9) influenza. J Infect Dis. 212:1214-1221. https://doi. org/10.1093/infdis/jiv246

17. Bizzotto J, Sanchis P, Abbate M, Lage-Vickers S, Lavignolle R, Toro A, Olszevicki S, Sabater A, Cascardo F, Vazquez E, Cotignola J, Gueron G (2020) SARS-CoV-2 Infection boosts MX1 antiviral effector in COVID-19 patients. iScience. 23:101585. https://doi. org/10.1016/j.isci.2020.101585

18. Vargas-Alarcón G, Posadas-Sánchez R, Ramírez-Bello J (2020) Variability in genes related to SARS-CoV-2 entry into host cells (ACE2, TMPRSS2, TMPRSS11A, ELANE, and CTSL) and its potential use in association studies. Life Sci. 260:118313. https:// doi.org/10.1016/j.lfs.2020.118313

19. Latini A, Agolini E, Novelli A, Borgiani P, Giannini R, Gravina P, Smarrazzo A, Dauri M, Andreoni M, Rogliani P, Bernardini S, Helmer-Citterich M, Biancolella M, Novelli G (2020) COVID-19 and genetic variants of protein involved in the SARS-CoV-2 entry into the host cells. Genes (Basel). 11:E1010. https://doi.org/10. 3390/genes 11091010

20. Pan D, Sze S, Minhas JS, Bangash MN, Pareek N, Divall P, Williams CML, Oggioni MR, Squire IB, Nellums LB, Hanif W, Khunti K, Pareek M (2020) The impact of ethnicity on clinical outcomes in COVID-19: a systematic review. EClinicalMedicine. https://doi.org/10.1016/j.eclinm.2020.100404

21. Cannalire R, Stefanelli I, Cerchia C, Beccari AR, Pelliccia S, Summa V (2020) SARS-CoV-2 Entry inhibitors: small molecules and peptides targeting virus or host cells. Int J Mol Sci. 21:5707. https://doi.org/10.3390/ijms21165707

22. Montopoli M, Zumerle S, Vettor R, Rugge M, Zorzi M, Catapano CV, Carbone GM, Cavalli A, Pagano F, Ragazzi E, Prayer-Galetti $\mathrm{T}$, Alimonti A (2020) Androgen-deprivation therapies for prostate cancer and risk of infection by SARS-CoV-2: a population-based study $(\mathrm{N}=4532)$. Ann Oncol. 31:1040-1045. https://doi.org/10. 1016/j.annonc.2020.04.479

23. Hassanpour M, Rezaie J, Nouri M, Panahi Y (2020) The role of extracellular vesicles in COVID-19 virus infection. Infect Genet Evol 85:104422. https://doi.org/10.1016/j.meegid.2020.104422

24. Gonzalez-Begne M, Lu B, Han X, Hagen FK, Hand AR, Melvin JE, Yates JR (2009) Proteomic analysis of human parotid gland exosomes by multidimensional protein identification technology (MudPIT). J Proteome Res 8:1304-1314. https://doi.org/10.1021/ pr800658c

Publisher's note Springer Nature remains neutral with regard to jurisdictional claims in published maps and institutional affiliations. 